\title{
Linking Contractive Self-Mappings and Cyclic Meir-Keeler Contractions with Kannan Self-Mappings
}

\author{
M. De la Sen \\ Instituto de Investigacion y Desarrollo de Procesos, Universidad del Pais Vasco, \\ Campus of Leioa (Bizkaia)—Aptdo, 644-Bilbao, 48080 Bilbao, Spain \\ Correspondence should be addressed to M. De la Sen, manuel.delasen@ehu.es
}

Received 1 September 2009; Accepted 22 February 2010

Academic Editor: Wataru Takahashi

Copyright (C) 2010 M. De la Sen. This is an open access article distributed under the Creative Commons Attribution License, which permits unrestricted use, distribution, and reproduction in any medium, provided the original work is properly cited.

Some mutual relations between $p$-cyclic contractive self-mappings, $p$-cyclic Kannan self-mappings, and Meir-Keeler $p$-cyclic contractions are stated. On the other hand, related results about the existence of the best proximity points and existence and uniqueness of fixed points are also formulated.

\section{Introduction}

In the last years, important attention is being devoted to extend the Fixed Point Theory by weakening the conditions on both the maps and the sets where those maps operate $[1,2]$. For instance, every nonexpansive self-mappings on weakly compact subsets of a metric space have fixed points if the weak fixed point property holds [1]. Further, increasing research interest relies on the generalization of Fixed Point Theory to more general spaces than the usual metric spaces such as, for instance, ordered or partially ordered spaces (see, e.g., [3-5]). Also, important fields of application of Fixed Point Theory exist nowadays in the investigation of the stability of complex continuous-time and discrete-time dynamic systems. The theory has been focused, in particular, on systems possessing internal lags, those being described by functional differential equations, those being characterized as hybrid dynamic systems and those being described by coupled continuous-time and discrete-time dynamics, [6-10]. On the other hand, Meir-Keeler self-mappings have received important attention in the context of Fixed Point Theory perhaps due to the associated relaxing in the required conditions for the existence of fixed points compared with the usual contractive mappings [11-14]. It also turns out from their definition that such self-mappings are less restrictive 
than strict contractive self-mappings so that their associated formalism is applicable to a wider class of real-life problems. Another interest of such self-mappings is their usefulness as a formal tool for the study of $p(\geq 2)$-cyclic contractions, even in the eventual case that the involved subsets of the metric space under study do not intersect, [12] so that there is no fixed point. In such a case, the usual role of fixed points is played by the best proximity points between adjacent subsets in the metric space. The underlying idea is that the best proximity points are fixed points if such subsets intersect while they play a close role to fixed points otherwise. On the other hand, there are also close links between contractive selfmappings and Kannan self-mappings [2,15-17] with constant $\alpha$ (referred to in the following as $\alpha$-Kannan self-mappings). In fact, $\alpha$-Kannan self-mappings are contractive for values of the contraction constant being less than $1 / 3$ [17].

The objective of this paper is to formulate some connections between $p$-cyclic contractive self-mappings, $p$-cyclic Meir-Keeler contractions, and $p$-cyclic $\alpha$-Kannan selfmappings. In particular, the existence and uniqueness of potential fixed points and also the best proximity points are investigated. The importance of cyclic maps in some problems as, for instance, in the case that a controlled state-solution trajectory of a dynamic system has to be driven from a set to its adjacent one in a certain time due to technical requirements, is well known. Consider a metric space $(X, d)$ and a self-mapping $T: A \cup B \rightarrow A \cup B$ such that $T(A) \subseteq B$ and $T(B) \subseteq A$ where $A$ and $B$ are nonempty subsets of $X$. Then, $T: A \cup B \rightarrow A \cup B$ is a 2-cyclic self-mapping what is said to be a 2-cyclic $k$-contraction self-mapping if it satisfies in addition

$$
d(T x, T y) \leq k d(x, y)+(1-k) \operatorname{dist}(A, B), \quad \forall x \in A, \forall y \in B
$$

for some real $k \in[0,1)$. The best proximity point is some $z \in A \cup B$ such that $d(z, T z)=$ $\operatorname{dist}(A, B)$. It turns out that if $A \cap B \neq \emptyset$, then $z \in F(T) \subset A \cup B$; that is, $z$ is a fixed point of $T$ since $\operatorname{dist}(A, B)=0$ [11-13]. If $k=1$, then $d(T x, T y) \leq d(x, y) ; \forall x \in A, \forall y \in B$ and $T: A \cup B \rightarrow A \cup B$ is a 2-cyclic nonexpansive self-mapping [12]. Nonexpansive mappings, in general, have received important attention in the last years. For instance, two hybrid methods are used in [18] to prove some strong convergence theorems. Those theorems are used to find a common element of the zero point set of a maximal monotone operator and the fixed point set of a relatively nonexpansive mapping in a Banach space. The concept of a strongly relatively nonexpansive sequence in a Banach space is given in [19]. The associate properties are investigated and applied approximating a common fixed point of a countable family of relatively nonexpansive mappings in uniformly convex and uniformly smooth Banach spaces. Also, the so-called $n$-times reasonable expansive and their properties self-mappings are investigated in [20].

\subsection{Notation}

$\mathbf{R}_{0+}:=\mathbf{R}_{+} \cup\{0\}, \mathbf{Z}_{0+}:=\mathbf{Z}_{+} \cup\{0\}, \mathbf{R}_{+}:=\{z \in \mathbf{R}: z>0\}$, and $\mathbf{Z}_{+}:=\{z \in \mathbf{Z}: z>0\}$ are the sets of nonnegative real and integer numbers and those of positive real and integer numbers, respectively,

$$
\bar{p}:=\{1,2, \ldots, p\} \subset \mathbf{Z}_{+} .
$$


$F(T)$ is the set of fixed points of a $p$-cyclic self-mapping $T$ in a nonempty subset $A$ of a metric space $(X, d)$.

$\mathrm{BP}_{i}(T)$ is the set of the best proximity points in a subset $A_{i}$ of a $p$-cyclic self-mapping $T$ on $\bigcup_{i=1}^{p} A_{i}$, namely, the union of a collection of nonempty subsets of a metric space $(X, d)$ which do not intersect.

Contractive self-mappings with constant $k$ and Kannan self-mappings with constant $\alpha$ are referred to as $k$-contractions and $\alpha$-Kannan self-mappings, respectively.

$\neg p$ (referred to as "not $p$ ") is the negation of a logic proposition $p$.

\section{About 2-Cyclic $k$-Contraction, 2-Cyclic $(\alpha, \beta)$-Kannan Self-Mappings, Contractions of Meir-Keeler Type, and Some Mutual Relationships}

The definition of $\alpha$-Kannan self-mappings $T: X \rightarrow X$ is as follows:

$$
d(T x, T y) \leq \alpha(d(x, T x)+d(y, T y)) ; \quad \forall x, y \in A \cup B
$$

for some real $\alpha \in[0,1 / 2)[14,15]$. Let us extend the above concept in a natural way to 2cyclic Kannan self-mappings by considering the definition of 2-cyclic $k$-contractions (1.1) as follows.

Definition 2.1. Consider a metric space $(X, d)$ and a self-mapping $T: A \cup B \rightarrow A \cup B$ such that $T(A) \subseteq B$ and $T(B) \subseteq A$ where $A$ and $B$ are nonempty subsets of $X$. Then $T: A \cup B \rightarrow A \cup B$ is a 2-cyclic $(\alpha, \beta)$-Kannan self-mapping for some real $\alpha \in[0,1 / 2)$ if it satisfies

$$
d(T x, T y) \leq \alpha(d(x, T x)+d(y, T y))+\beta(1-\alpha) \operatorname{dist}(A, B) ; \quad \forall x \in A, \forall y \in B
$$

for some $\beta \in \mathbf{R}_{+}$. Definition 2.1 is a natural counterpart of (2.1) for $\alpha$-Kannan self-mappings by taking into account the definition of a 2-cyclic $k$-contraction in (1.1).

Remark 2.2. Let $(X, d)$ be a metric space and let $T: A \cup B \rightarrow A \cup B$ be a 2-cyclic $k$-contraction self-mapping with $A$ and $B$ being nonempty nondisjoint subsets of $X$. It turns out that $T$ : $A \cup B \rightarrow A \cup B$ is also a contractive mapping with constant $k$.

Contraction self-mappings can also be 2-cyclic $\alpha$-Kannan self-mappings and vice-versa as addressed in the two following results:

Proposition 2.3. Assume that $T: A \cup B \rightarrow A \cup B$ is a 2-cyclic $k$-contraction self-mapping with $k \in[0,1 / 3)$. Then $T: A \cup B \rightarrow A \cup B$ is also a 2-cyclic $(k /(1-k), \beta)$-Kannan self-mapping, $\forall \beta \geq \beta_{0}:=(1-k) /(1-2 k)$. 
Proof. The following inequalities follow from (1.1) and the triangle inequality of the distance map $d: X \times X \rightarrow \mathbf{R}_{0_{+}}$:

$$
\begin{aligned}
d(T x, T y) \leq & k d(x, y)+(1-k) \operatorname{dist}(A, B) \\
\leq & k(d(x, T x)+d(y, T y))+k d(T x, T y) \\
& +(1-k) \operatorname{dist}(A, B) ; \quad \forall x \in A, \forall y \in B,
\end{aligned}
$$

for some $k \in[0,1 / 3)$, what leads to

$$
\begin{aligned}
d(T x, T y) & \leq \frac{k}{1-k}(d(x, T x)+d(y, T y))+\operatorname{dist}(A, B) \\
& \leq \frac{k}{1-k}(d(x, T x)+d(y, T y))+\beta\left(\frac{1-2 k}{1-k}\right) \operatorname{dist}(A, B) ; \quad \forall x \in A, \forall y \in B
\end{aligned}
$$

for $\beta((1-2 k) /(1-k)) \geq 1, \forall k \in[0,1 / 2), \forall \beta \geq \beta_{0}:=(1-k) /(1-2 k)$. Thus, $T: A \cup B \rightarrow$ $A \cup B$ is a 2-cyclic $(k /(1-k), \beta)$-Kannan self-mapping from Definition 2.1 for $\beta \in\left[\beta_{0}, \infty\right)$ if $\alpha=k /(1-k) \in[0,1 / 2) \Leftrightarrow k \in[0,1 / 3)$. The proof is complete.

Proposition 2.4. Assume that $A$ and $B$ are closed disjoint nonempty bounded simply connected sets with diam $A \leq K_{A} \operatorname{dist}(A, B)$ and $\operatorname{diam} B \leq K_{B} \operatorname{dist}(A, B)$ for some $K_{A} \in \mathbf{R}_{+}, K_{B} \in \mathbf{R}_{+}$. Assume also that $T: A \cup B \rightarrow A \cup B$ is a 2-cyclic $(\alpha, \beta)$-Kannan self-mapping with $\alpha$ and $\beta$ subject to the constraints $\alpha \leq \max \left(1 / K_{A B}, 1 / 2\right)$ and $\beta \leq\left(1-\alpha K_{A B}\right) /(1-\alpha)$ with $K_{A B}:=1+\min \left(K_{A}+\right.$ $\left.2 K_{B}, K_{B}+2 K_{A}\right)$. Then, $T: A \cup B \rightarrow A \cup B$ is also a 2-cyclic $k$-contraction self-mapping for any real constant $k \leq 1-\alpha K_{A B}-\beta(1-\alpha)$.

Proof. Since $T: A \cup B \rightarrow A \cup B$ is a 2-cyclic $(\alpha, \beta)$-Kannan self-mapping then from Definition 2.1, it follows that

$$
\begin{aligned}
d(T x, T y) \leq & \alpha(d(x, T x)+d(y, T y))+\beta(1-\alpha) \operatorname{dist}(A, B) \\
\leq & \alpha(d(x, y)+d(y, T x)+d(y, T y)) \\
& +\beta(1-\alpha) \operatorname{dist}(A, B) \quad \forall x \in A, \forall y \in B
\end{aligned}
$$

Also, one has that $d(y, T x) \leq \operatorname{diam}(B), \forall x \in A, \forall y \in B$, since $T x \in B ; \forall x \in A$. Choosing $x_{0} \in A$ and $y_{0} \in B$ such that $d\left(x_{0}, y_{0}\right)=\operatorname{dist}(A, B)$, the triangle inequality for the distance map yields

$$
\begin{aligned}
\alpha d(y, T y) & \leq \alpha\left(d\left(y, y_{0}\right)+d\left(y_{0}, x_{0}\right)+d\left(x_{0}, x\right)\right) \\
& \leq \alpha(\operatorname{diam}(A)+\operatorname{diam}(B)+\operatorname{dist}(A, B)) \\
& \leq \alpha\left(K_{A}+K_{B}+1\right) \operatorname{dist}(A, B)
\end{aligned}
$$


so that one has since $K_{A B} \geq K_{A}+2 K_{B}+1$ :

$$
\begin{aligned}
& \alpha(d(y, T x)+d(y, T y))+\beta(1-\alpha) \operatorname{dist}(A, B) \\
& \quad \leq\left(\alpha K_{A B}+\beta(1-\alpha)\right) \operatorname{dist}(A, B) \leq \alpha \operatorname{dist}(A, B) \leq(1-k) \operatorname{dist}(A, B)
\end{aligned}
$$

provided that $k \leq 1-\alpha K_{A B}-\beta(1-\alpha)$ under the necessary conditions $\beta \leq\left(1-\alpha K_{A B}\right) /(1-\alpha)$ and $\alpha \leq \max \left(1 / K_{A B}, 1 / 2\right)$. The above derivation remains valid by interchanging the roles of the sets $A$ and $B$ since $K_{A B} \geq K_{B}+2 K_{A}+1$.

The following two results are direct.

Corollary 2.5. Proposition 2.4 holds "mutatis-mutandis" if either A or B is an open set.

Proof. It follows under the same reasoning by taking $x_{0}$ in $\operatorname{cl} A$ if $A$ is open and $y_{0}$ in $\operatorname{cl} B$ if $B$ is open.

Corollary 2.6. Proposition 2.4 and Corollary 2.5 cannot be fulfilled for any $(x, y) \in A \times B$ if $A \cap B \neq \emptyset$.

Proof. It is direct since $A \cap B=\emptyset \Rightarrow \operatorname{dist}(A, B)=0$ and $K_{A}$ and $K_{B}$ are infinite.

Definition 2.7 (see [12]). A 2-cyclic contraction self-mapping $T: A \cup B \rightarrow A \cup B$ is of MeirKeeler type if for any given $\varepsilon \in \mathbf{R}_{+}, \exists \delta=\delta(\varepsilon) \in \mathbf{R}_{+}$such that

$$
d(x, y)<\varepsilon+\delta+\operatorname{dist}(A, B) \Longrightarrow d(T x, T y)<\varepsilon+\operatorname{dist}(A, B) ; \quad \forall(x, y) \in A \times B
$$

Keeler type

The subsequent result is concerned with 2-cyclic contraction self-mappings of Meir-

Proposition 2.8. Assume that $T: A \cup B \rightarrow A \cup B$ is a 2-cyclic contraction self-mapping of MeirKeeler type. Then, the following properties hold:

(i) If $A \cap B \neq \emptyset$, then $F(T)(\neq \emptyset) \subset A \cap B$ and $\operatorname{dist}(A, B)=d(z, T z) ; \forall z \in F(T)$, that is, all the best proximity points are also fixed points.

(ii) If $A \cap B=\emptyset$, then either $\neg \exists \lim _{j \rightarrow \infty} d\left(T^{j} x, T^{j} y\right)$ or $\exists \lim _{j \rightarrow \infty} d\left(T^{j} x, T^{j} y\right)>0 ; \forall(x, y) \in$ $A \times B$.

Also, $\exists \rho=\rho(\varepsilon) \in \mathbf{R}_{+}$such that

$$
\limsup _{j \rightarrow \infty} d\left(T^{j} x, T^{j} y\right)<\rho \varepsilon+\operatorname{dist}(A, B) ; \quad \forall(x, y) \in A \times B \text { for some } \rho=\rho(\varepsilon) \in \mathbf{R}
$$

Proof. Note from (2.8) that

$$
d\left(T^{\ell} x, T^{\ell} y\right)<\varepsilon+\delta_{\ell}+\operatorname{dist}(A, B) \Longrightarrow d\left(T^{\ell+1} x, T^{\ell+1} y\right)<\varepsilon+d\left(T^{\ell} x, T^{\ell} y\right) ; \quad \forall(x, y) \in A \times B
$$


for some $\delta_{\ell}=\delta_{\ell}(\varepsilon) \in \mathbf{R}_{+} ; \forall \ell \in \mathbf{Z}_{0+}$. Define $\mathbf{R}_{+} \ni M_{j}=M_{j}\left(\varepsilon, \delta_{j}\right):=\delta_{j} / \varepsilon ; \forall j \in \mathbf{Z}_{+}$. Since $T$ : $A \cup B \rightarrow A \cup B$ is a 2-cyclic contraction self-mapping of Meir-Keeler type, one has proceeding recursively with (2.10)

$$
\begin{aligned}
d(x, y) & <\varepsilon+\delta_{0}+\operatorname{dist}(A, B) \Longrightarrow d\left(T^{j+1} x, T^{j+1} y\right) \\
& <\left(1-\sum_{\ell=1}^{j} M_{\ell}\right) \varepsilon+\operatorname{dist}(A, B) ; \quad \forall(x, y) \in A \times B,
\end{aligned}
$$

so that (2.10) together with the constraint $d\left(T^{j+1} x, T^{j+1} y\right) \geq 0 ; \forall j \in \mathbf{Z}_{0+}$ implies that

$$
\left(1-\sum_{\ell=1}^{j} M_{\ell}\right) \varepsilon+\operatorname{dist}(A, B) \geq 0 \Longrightarrow M_{\ell} \longrightarrow 0 \quad \text { as } \ell \longrightarrow \infty
$$

If, in addition, $\operatorname{dist}(A, B)=0$, that is, $A \cap B \neq \emptyset$, then $\limsup _{j \rightarrow \infty} \sum_{\ell=1}^{j} M_{\ell} \leq 1$ (otherwise, $0 \leq \limsup _{j \rightarrow \infty} d\left(T^{j+1} x, T^{j+1} y\right)<0 ; \forall(x, y) \in A \times B$ would be a contradiction), so that $\exists \lim _{j \rightarrow \infty} d\left(T^{j} x, T^{j} y\right)=0$ and $\exists z \in F(T)(\neq \emptyset) \subset A \cap B \subset A \cup B$ such that $\operatorname{dist}(A, B)=d(z, T z)$ which are the best proximity points and also fixed points.

If $A \cap B=\emptyset$, then $\operatorname{dist}(A, B)=d(z, T z)>0$ for some $\exists z \in A \cup B$ which is not obviously a fixed point, since $A \cap B=\emptyset$, so that

$$
\limsup _{j \rightarrow \infty} d\left(T^{j} x, T^{j} y\right)<\rho \varepsilon+\operatorname{dist}(A, B) ; \quad \forall(x, y) \in A \times B
$$

for some $\rho=\rho(\varepsilon) \in \mathbf{R}$ defined by $\rho:=\lim _{\inf _{j \rightarrow \infty}}\left(1-\sum_{\ell=1}^{j} M_{\ell}\right) \geq-\operatorname{dist}(A, B) / \varepsilon$. Furthermore, $\neg \exists \lim _{j \rightarrow \infty} d\left(T^{j} x, T^{j} y\right)$ since $\left(T^{j} x, T^{j} y\right) \in A \times B$ (resp., $\left(T^{j} x, T^{j} y\right) \in B \times A$ ), what yields $\left(T^{j+1} x, T^{j+1} y\right) \in A \times B$ (resp., $\left.\left(T^{j+1} x, T^{j+1} y\right) \in A \times B\right) ; \forall j \in \mathbf{Z}_{0+}$ and $A \times B \cap B \times A=\emptyset$ since $A \cap B=\emptyset$.

Definition 2.9. A 2-cyclic $(\alpha, \beta)$-Kannan self-mapping $T: A \cup B \rightarrow A \cup B$ defined for some real $\alpha \in[0,1 / 2)$ and some $\beta \in \mathbf{R}_{+}$(see Definitions 2.1 and 2.7) is of Meir-Keeler type if for any given $\varepsilon \in \mathbf{R}_{+}, \exists \delta=\delta(\varepsilon) \in \mathbf{R}_{+}$such that (2.8) holds. result.

Proposition 2.3, Definitions 2.1 and 2.9, and Proposition 2.8 yield directly the following

Proposition 2.10. Assume that $T: A \cup B \rightarrow A \cup B$ is a 2-cyclic contraction self-mapping of MeirKeeler type with $k \in[0,1 / 3)$. Then, $T: A \cup B \rightarrow A \cup B$ is also a 2-cyclic $(k /(1-k), \beta)$-Kannan self-mapping, $\forall \beta \geq \beta_{0}:=(1-k) /(1-2 k)$. If $A \cap B \neq \emptyset$, then $F(T)(\neq \emptyset) \subset A \cap B$.

From the definition of $k$-contraction self-mappings and Definition 2.7 for Meir-Keeler type contraction self-mappings, the following result holds. 
Fixed Point Theory and Applications

Proposition 2.11. If $T: A \cup B \rightarrow A \cup B$ is a k-contraction self-mapping of Meir-Keeler type, then for any given $\varepsilon \in \mathbf{R}_{+}, \exists \delta=\delta(\varepsilon) \in \mathbf{R}_{+}$and $\varepsilon_{0}=\varepsilon_{0}(\varepsilon) \in[0, \varepsilon) \cap \mathbf{R}$ such that

$$
d(T x, T y) \leq \operatorname{dist}(A, B)+\min \left(\varepsilon_{0}, k(d(x, y)-\operatorname{dist}(A, B))\right) ; \quad \forall(x, y) \in A \times B
$$

Proof. Since $T: A \cup B \rightarrow A \cup B$ is a contraction self-mapping which is also of Meir-Keeler type, the result follows directly by combining (1.1) and (2.8).

\section{3. $p$-Cyclic $k$-Contraction, Contractions of Meir-Keeler Type, $p$-Cyclic $(\alpha, \beta)$-Kannan Self-Mappings, and Some Mutual Relationships}

A set of relevant results for $p$-cyclic self-mappings for $p>2$ are obtained in [12]. Those selfmappings obey the subsequent definitions.

Definition 3.1 (see [12]). Let $A_{i}$ be nonempty subsets of a metric space $(X, d) ; \forall i \in \bar{p}$. Then, $T: \bigcup_{i=1}^{p} A_{i} \rightarrow \bigcup_{i=1}^{p} A_{i}$ is a $p$-cyclic self-mapping if $T\left(A_{i}\right) \subseteq A_{i+1} ; \forall i \in \bar{p}$ with $A_{p+j}=A_{j}$; $\forall j \in \mathbf{Z}_{+}$.

Definition 3.2. Let $A_{i}$ be nonempty, $\forall i \in \bar{p}$ subsets of a metric space $(X, d)$. Then, $T: \bigcup_{i=1}^{p} A_{i} \rightarrow$ $\bigcup_{i=1}^{p} A_{i}$ is a $p$-cyclic $k$-contraction self-mapping if $T\left(A_{i}\right) \subseteq A_{i+1} ; \forall i \in \bar{p}$ with $A_{p+j}=A_{j} ; \forall j \in \mathbf{Z}_{+}$ and, furthermore,

$$
d(T x, T y) \leq k d(x, y)+(1-k) \operatorname{dist}\left(A_{i}, A_{i+1}\right) ; \quad \forall x \in A_{i}, \forall y \in A_{i+1}
$$

for some real constant $k \in[0,1)$.

A point $x \in A_{i}$ is said to be the best proximity point if $d(x, T x)=\operatorname{dist}\left(A_{i}, A_{i+1}\right)$, [12]. In this paper, it is also proven that if $T: \bigcup_{i=1}^{p} A_{i} \rightarrow \bigcup_{i=1}^{p} A_{i}$ is a $p$-cyclic nonexpansive selfmapping, that is, $d(T x, T y) \leq d(x, y) ; \forall(x, y) \in A_{i} \times A_{i+1} ; \forall i \in \bar{p}$, then $\operatorname{dist}\left(A_{i}, A_{i+1}\right)=d_{A} \in$ $\mathbf{R}_{0+} ; \forall i \in \bar{p}$ (i.e., the distances between adjacent sets are identical). Some properties concerned with $p$-cyclic nonexpansive self-mappings are stated and proven in the next lemma.

Lemma 3.3. The following properties hold:

(i) let $T: \bigcup_{i=1}^{p} A_{i} \rightarrow \bigcup_{i=1}^{p} A_{i}$ be a $p$-cyclic $k$-contraction self-mapping, then,

$$
\begin{gathered}
d\left(T^{j} x, T^{j} y\right) \leq k^{j} d(x, y)+(1-k) j d_{A}, \\
\forall(x, y) \in A_{i} \times A_{i+1} ; \quad \forall i \in \bar{p}, \forall j \in \mathbf{Z}_{0+}, \\
d\left(T^{i p+\ell} x, T^{i p+\ell} y\right) \leq k^{i p+\ell} d(x, y)+(1-k)(i p+\ell) d_{A} \\
\forall(x, y) \in A_{i} \times A_{i+1}, \quad \forall \ell \in \overline{p-1} \cup\{0\}, \quad \forall i \in \bar{p}, \forall j \in \mathbf{Z}_{0+}
\end{gathered}
$$

where $d_{A}:=\operatorname{dist}\left(A_{i}, A_{i+1}\right) ; \forall i \in \bar{p}$. 
Let the mappings $\mathbf{T}(j, i): A_{i} \cup A_{i+1} \rightarrow A_{j} \cup A_{j+1} ; \forall j \in \bar{p} \backslash \bar{i}, \forall i \in \bar{p}$, be defined by

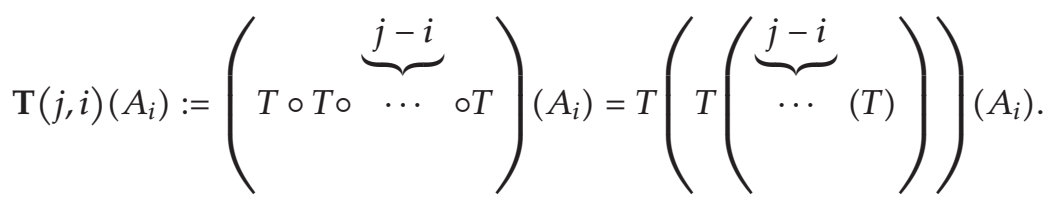

If $\bigcap_{i=1}^{p} \mathbf{T}(j, i)\left(A_{i}\right) \neq \emptyset ; \forall j \in \bar{p} \backslash \bar{i}, \forall i \in \bar{p}$, with $\mathbf{T}(i+1, i)=T ; \forall i \in \bar{p}$, there is $z \in F(T)(\neq \emptyset)$ which is unique if $(X, d)$ is complete.

(ii) If $T: \bigcup_{i=1}^{p} A_{i} \rightarrow \bigcup_{i=1}^{p} A_{i}$ is a $p$-cyclic nonexpansive self-mapping and, in particular, if t is a p-cyclic $k$-contraction, then

$$
d\left(T^{j} x, T^{j} y\right) \leq d(x, y) ; \quad \forall(x, y) \in A_{i} \times A_{i+1} ; \forall j \in \mathbf{Z}_{0+}, \forall i \in \bar{p}
$$

Proof. (i) Equation (3.2) follows by constructing a recursion directly from (1.1); $\forall(x, y) \in$ $A_{i} \times A_{i+1} ; \forall i \in \bar{p}, \forall j \in \mathbf{Z}_{0+}$ which can be also written equivalently in the form (3.3) by using the index identity $j=i p+\ell ; \forall i \in \bar{p}, \forall \ell \in \overline{p-1} \cup\{0\}$. If $\bigcap_{i=1}^{p} \mathbf{T}(j, i)\left(A_{i}\right) \neq \emptyset \Rightarrow \bigcap_{i=1}^{p} A_{i} \neq \emptyset \Rightarrow$ $d_{A}=0$, then $\lim _{j \rightarrow \infty} d\left(T^{j} x, T^{j} y\right) \leq \lim _{j \rightarrow \infty} k^{j} d(x, y)=0 ; \forall(x, y) \in\left(A_{i}, A_{i+1}\right), \forall i \in \bar{p}$ from (3.2) since $k \in[0,1)$, so that there exists $\lim _{j \rightarrow \infty} T^{j} x=z \in\left(\bigcap_{i=1}^{p} \mathbf{T}(p+i, i)\right) ; \forall x \in \bigcup_{i=1}^{p} A_{i}$. The point $z$ is in $F(T) \neq \emptyset$ since $F(T) \supseteq F\left(\bigcap_{i=1}^{p} \mathbf{T}(p+i, i)\right) \neq \emptyset$ by construction of the self-mappings $\mathbf{T}(j, i): A_{i} \cup A_{i+1} \rightarrow A_{j} \cup A_{j+1} ; \forall j \in \bar{p} \backslash \bar{i}, \forall i \in \bar{p}$ since $\bigcap_{i=1}^{p} \mathbf{T}(p+i, i) \neq \emptyset$. Also, $z$ is unique if $(X, d)$ is complete. Property (i) has been proven.

(ii) It follows from the recursion $d\left(T^{j} x, T^{j} y\right) \leq \cdots \leq d(T x, T y) \leq d(x, y) \forall(x, y) \in A_{i} \times$ $A_{i+1} ; \forall j \in \mathbf{Z}_{0+}$, obtained from (3.2) for $k=1$, since $T: \bigcup_{i=1}^{p} A_{i} \rightarrow \bigcup_{i=1}^{p} A_{i}$ is nonexpansive.

Lemma 3.3.

The auxiliary properties of Remark 3.4 below have been used in the proof of

Remark 3.4. Note that

$$
\bigcap_{i=1}^{p} \mathbf{T}(p+i, i)\left(A_{i}\right)=\bigcap_{j \in \bar{p} \backslash \bar{i}} \bigcap_{i=1}^{p} \mathbf{T}(j, i)\left(A_{i}\right),
$$

so that

$$
\begin{gathered}
F(T) \supseteq F(\mathbf{T}(p+i, i)) \supseteq F\left(\bigcap_{i=1}^{p} \mathbf{T}(p+i, i)\right) ; \quad \forall i \in \bar{p}, \\
\bigcap_{j \in \bar{p} \backslash \bar{i}} \bigcap_{i=1}^{p} \mathbf{T}(j, i)\left(A_{i}\right) \subseteq \bigcap_{i=1}^{p} T\left(A_{i}\right) \subseteq \bigcap_{i=1}^{p} A_{i},
\end{gathered}
$$

so that

$$
\bigcap_{i=1}^{p} \mathbf{T}(p+i, i)\left(A_{i}\right) \neq \emptyset \Longrightarrow \bigcap_{i=1}^{p} T\left(A_{i}\right) \neq \emptyset \Longrightarrow \bigcap_{i=1}^{p} A_{i} \neq \emptyset
$$


The concepts of $p$-cyclic nonexpansive self-mapping and $p$-cyclic $k$-contraction are generalized in the following. Consider the mappings $T_{i}: A_{i} \cup A_{i+1} \rightarrow A_{i+1} \cup A_{i+2}$ with $T_{i}\left(A_{j}\right) \subseteq A_{j+1} ; j=i, i+1, \forall i \in \bar{p}$ and $A_{p+j}=A_{j} ; \forall j \in \mathbf{Z}_{+}$which fulfil the constraint

$$
d\left(T_{i} x, T_{i} y\right) \leq k_{i} d(x, y)+\left|1-k_{i}\right| \operatorname{dist}\left(A_{i}, A_{i+1}\right) ; \quad \forall x \in A_{i}, \forall y \in A_{i+1} ; \forall i \in \bar{p}
$$

for some $k_{i} \in \mathbf{R}_{+} ; \forall i \in \bar{p}$ and associated composed mappings $\mathbf{T}(j, i): A_{i} \cup A_{i+1} \rightarrow A_{j} \cup A_{j+1}$ defined by $\mathbf{T}(j, i):=T_{j} \circ T_{j-1} \circ \cdots \circ T_{i+1} \circ T_{i} ; \forall i, j(>i) \in \bar{p}$, subject to $1 \leq j-i \leq p$.

Remark 3.5. Note that $\mathbf{T}(p+i, i): A_{i} \cup A_{i+1} \rightarrow A_{i} \cup A_{i+1}$ is also defined for points $x \in \bigcap_{j \in I_{i}} A_{j}$ for some nonempty indexing set $I_{i} \subset \bar{p}$, which contains $i \in \bar{p}$, by restricting its domain and image as $T_{i}: \bigcap_{j \in I_{i}} A_{j} \rightarrow \bigcap_{j \in I_{i+1}} A_{j}$ for some nonempty indexing set $I_{i+1} \subset \bar{p}$ such that $T_{i} x \in$ $\bigcap_{j \in I_{i+1}} A_{j} \subset A_{i+1}$ (since $x \in \bigcap_{j \in I_{i}} A_{j} \subset A_{i}$ ). An important observation is that a set of constraints of type (3.9) have to be satisfied if $\operatorname{card}\left(I_{i}\right)>1$

$$
\begin{aligned}
& d\left(T_{j} x, T_{j} y\right) \leq k_{j} d(x, y)+\left|1-k_{j}\right| \operatorname{dist}\left(A_{j}, A_{j+1}\right) \\
& \quad \forall x \in \bigcap_{j \in I_{i}} A_{j}, \quad \forall y \in \bigcap_{j \in I_{i+1}} A_{j} ; \quad \forall j \in I_{i} \text { for each } i \in \bar{p} .
\end{aligned}
$$

The subsequent definitions extend Definitions 3.1-3.2 by removing the necessity of the set inclusions $T\left(A_{i}\right) \subseteq T\left(A_{i+1}\right) ; \forall i \in \bar{p}$ and allowing obtaining of contractions from the composed mappings $T_{i}: A_{i} \cup A_{i+1} \rightarrow A_{i+1} \cup A_{i+2} ; \forall i \in \bar{p}$ which are not all necessarily contractions provided that $A_{i} \subseteq A_{i+1} ; \forall i \in \bar{p}$.

Definition 3.6. Let $A_{i}$ be nonempty subsets of a metric space with $A_{p+i}=A_{i}, \forall i \in \bar{p}$. Then, $\mathbf{T}(p+i, i): A_{i} \cup A_{i+1} \rightarrow A_{i} \cup A_{i+1} ; \forall i \in \bar{p}$ is a composed $p$-cyclic self-mapping if $T\left(A_{i}\right) \subseteq A_{i+1}$; $\forall i \in \bar{p}$ with $A_{p+j}=A_{j} ; \forall j \in \mathbf{Z}_{+}$.

Definition 3.7. Let $A_{i}$ be nonempty subsets of a metric space $(X, d)$ with $A_{p+i}=A_{i}, \forall i \in \bar{p}$. Then, $\mathbf{T}(p+i, i): A_{i} \cup A_{i+1} \rightarrow A_{i} \cup A_{i+1} ; i \in \bar{p}$ is a composed $p$-cyclic $k$-contraction selfmapping if $T_{i}: A_{i} \cup A_{i+1} \rightarrow A_{i+1} \cup A_{i+2}$ satisfies (3.9), subject to $T_{i}\left(A_{j}\right) \subseteq A_{j+1}, j=i, i+1$, and, furthermore,

$$
d(\mathbf{T}(p+i, i) x, \mathbf{T}(p+i, i) y) \leq k d(x, y)+(1-k) \operatorname{dist}\left(A_{i}, A_{i+1}\right) ; \quad \forall x \in A_{i}, \forall y \in A_{i+1}
$$

for some real constant $k \in[0,1)$ and, furthermore, if $T\left(A_{i}\right) \subseteq A_{i+1} ; \forall i \in \bar{p}$ with $A_{p+j}=A_{j}$; $\forall j \in \mathbf{Z}_{+}$.

Definition 3.8. If $\mathbf{T}(p+i, i): A_{i} \cup A_{i+1} \rightarrow A_{i} \cup A_{i+1}$ fulfils Definition 3.7 with (3.11) being true also for $k=1$, then it is said to be a composed $p$-cyclic nonexpansive mapping.

Note that if $\mathbf{T}(p+i, i): A_{i} \cup A_{i+1} \rightarrow A_{i} \cup A_{i+1}$ is a composed $p$-cyclic nonexpansive self-mapping (resp., a composed $p$-cyclic $k$-contraction self-mapping) for some $i \in \bar{p}$ then it is so for all $i \in \bar{p}$. Composed $p$-cyclic contractions are characterized according to tests stated and proven in the subsequent result. 
Proposition 3.9. Assume that $A_{i}$ are nonempty subsets of a metric space $(X, d) ; \forall i \in \bar{p}$. Assume also that $T_{i}\left(A_{j}\right) \subseteq A_{j+1} ; j=i, i+1 ; \forall i \in \bar{p}$ and that $\mathbf{T}(p+i, i): A_{i} \cup A_{i+1} \rightarrow A_{i} \cup A_{i+1} ; \forall i \in \bar{p}$ fulfils (3.9) for some $k_{i} \in \mathbf{R}_{+} ; \forall i \in \bar{p}$.

Then, the self-mapping $\mathbf{T}(p+i, i): A_{i} \cup A_{i+1} \rightarrow A_{i} \cup A_{i+1} ; i \in \bar{p}$ is a composed $p$-cyclic $k$-contraction self-mapping if the following two conditions hold:

$$
\begin{gathered}
k:=\prod_{i=1}^{p}\left[k_{i}\right]<1, \\
\operatorname{dist}\left(A_{i}, A_{i+1}\right) \geq \frac{\sum_{\ell(\neq i)=1}^{p-1}\left(\prod_{j=\ell+1}^{p-1}\left[k_{j}\right]\right)\left|1-k_{\ell}\right| \operatorname{dist}\left(A_{\ell}, A_{\ell+1}\right)}{\left(1-\prod_{j=1}^{p}\left[k_{j}\right]\left(1+\left|1-k_{i}\right|\right)\right)} \text { if } \prod_{j=1}^{p}\left[k_{j}\right]\left(1+\left|1-k_{i}\right|\right) \neq 1
\end{gathered}
$$

or $A_{\ell} \subseteq A_{\ell+1} ; \forall \ell \in \bar{p}$, otherwise.

If $k<1$ and $A_{\ell} \subseteq A_{\ell+1} ; \forall \ell \in \bar{p}$, then $\mathrm{T}(p+i, i): A_{i} \cup A_{i+1} \rightarrow A_{i} \cup A_{i+1} ; i \in \bar{p}$ is a composed $p$-cyclic $k$-contraction self-mapping.

Proof. If $\mathbf{T}(p+i, i): A_{i} \cup A_{i+1} \rightarrow A_{i} \cup A_{i+1} ; \forall i \in \bar{p}$ fulfils (3.9), then for any $i \in \bar{p}$

$$
\begin{array}{r}
d(\mathbf{T}(p+i, i) x, \mathbf{T}(p+i, i) y) \leq \prod_{j=i}^{p+i}\left[k_{j}\right] d(x, y)+\sum_{\ell=i}^{p+i}\left(\prod_{j=\ell+1}^{p+i}\left[k_{j}\right]\right)\left|1-k_{\ell}\right| \operatorname{dist}\left(A_{\ell}, A_{\ell+1}\right) \\
\forall x \in A_{i}, \quad \forall y \in A_{i+1},
\end{array}
$$

since $k_{i+p}=k_{i} ; A_{i+p}=A_{i} ; \forall i \in \bar{p}$. Then, $\mathbf{T}(p+i, i): A_{i} \cup A_{i+1} \rightarrow A_{i} \cup A_{i+1}$ is a composed $p$-cyclic $k$-contraction self-mapping from (3.12) and (3.11) (see Definition 3.7) if

$$
\begin{array}{r}
\prod_{j=i}^{p+i}\left[k_{j}\right]<1, \quad \sum_{\ell=i}^{p+i}\left(\prod_{j=\ell+1}^{p+i}\left[k_{j}\right]\right)\left|1-k_{\ell}\right| \operatorname{dist}\left(A_{\ell}, A_{\ell+1}\right) \leq\left(1-\prod_{j=1}^{p}\left[k_{j}\right]\right) \operatorname{dist}\left(A_{i}, A_{i+1}\right) \\
\forall x \in A_{i}, \quad \forall y \in A_{i+1},
\end{array}
$$


since $k_{i+p}=k_{i} ; \forall i \in \bar{p}$. The second inequality of (3.13) is equivalent to

$$
\begin{aligned}
\sum_{\ell=i+1}^{p+i}\left(\prod_{j=\ell+1}^{p+i}\left[k_{j}\right]\right)\left|1-k_{\ell}\right| \operatorname{dist}\left(A_{\ell}, A_{\ell+1}\right)+\left(\prod_{j=i+1}^{p+i}\left[k_{j}\right]\right)\left|1-k_{i}\right| \operatorname{dist}\left(A_{i}, A_{i+1}\right) \\
\leq\left(1-\prod_{j=1}^{p}\left[k_{j}\right]\right) \operatorname{dist}\left(A_{i}, A_{i+1}\right) \\
\Longleftrightarrow \sum_{\ell=i+1}^{p+i}\left(\prod_{j=\ell+1}^{p+i}\left[k_{j}\right]\right)\left|1-k_{\ell}\right| \operatorname{dist}\left(A_{\ell}, A_{\ell+1}\right) \\
\leq\left(\left(1-\prod_{j=1}^{p}\left[k_{j}\right]\right)-\left(\prod_{j=i+1}^{p+i}\left[k_{j}\right]\right)\left|1-k_{i}\right|\right) \operatorname{dist}\left(A_{i}, A_{i+1}\right) .
\end{aligned}
$$

Again since $k_{i+p}=k_{i} ; \forall i \in \bar{p}$ and since $A_{i+p}=A_{i} ; \forall i \in \bar{p}$, then $\prod_{j=i+1}^{p+i}\left[k_{j}\right]=\prod_{j=1}^{p}\left[k_{j}\right]$, and

$$
\sum_{\ell=i+1}^{p+i-1}\left(\prod_{j=\ell+1}^{p+i-1}\left[k_{j}\right]\right)\left|1-k_{\ell}\right| \operatorname{dist}\left(A_{\ell}, A_{\ell+1}\right)=\sum_{\ell(\neq i)=1}^{p}\left(\prod_{j=\ell+1}^{p}\left[k_{j}\right]\right)\left|1-k_{\ell}\right| \operatorname{dist}\left(A_{\ell}, A_{\ell+1}\right) .
$$

Then (3.15) and (3.14), are equivalent to

$$
\sum_{\ell(\neq i)=1}^{p-1}\left(\prod_{j=\ell+1}^{p-1}\left[k_{j}\right]\right)\left|1-k_{\ell}\right| \operatorname{dist}\left(A_{\ell}, A_{\ell+1}\right) \leq\left(1-\prod_{j=1}^{p}\left[k_{j}\right]\left(1+\left|1-k_{i}\right|\right)\right) \operatorname{dist}\left(A_{i}, A_{i+1}\right) .
$$

The first part of the result has been proven since (3.11) holds. The second one is a direct conclusion of the first one for the case $\operatorname{dist}\left(A_{\ell}, A_{\ell+1}\right)=0$.

It is now proven that if $\prod_{i=1}^{p}\left[k_{i}\right]<1$ and $A_{i} \subseteq A_{i+1} ; \forall i \in \bar{p}$, then all the self-mappings $\mathrm{T}(p+i, i): A_{i} \cup A_{i+1} \rightarrow A_{i} \cup A_{i+1} ; \forall i \in \bar{p}$ are composed $p$-cyclic $k$-contraction self-mappings possessing fixed points. If, furthermore, $(X, d)$ is a complete metric space, then each of those self-mappings possesses a unique fixed point.

Corollary 3.10. Assume that $A_{i}$ are nonempty subsets of a metric space $(X, d) ; \forall i \in \bar{p}$ and the composed $p$-cyclic $k$-contraction self-mapping $\mathbf{T}(p+i, i): A_{i} \cup A_{i+1} \rightarrow A_{i} \cup A_{i+1}$ fulfils Proposition 3.9 for some $i \in \bar{p}$, subject to $T_{i}\left(A_{j}\right) \subseteq A_{j+1} ; j=i, i+1 ; \forall i \in \bar{p}$. Then, the following properties hold provided that $A_{i} \subseteq A_{i+1} ; \forall i \in \bar{p}$.

(i) $\mathbf{T}(p+i, i): A_{i} \cup A_{i+1} \rightarrow A_{i} \cup A_{i+1} ; \forall i \in \bar{p}$ are all composed $p$-cyclic $k$-contraction selfmappings which satisfy, in addition, $A_{i} \subseteq A_{i+1} ; \forall i \in \bar{p}$ (i.e., $k:=\prod_{i=1}^{p}\left[k_{i}\right]<1$ and $\operatorname{dist}\left(A_{i}, A_{i+1}\right)=0$; $\forall i \in \bar{p})$ and which possess common fixed points in $\bigcap_{j=1}^{p} T_{j}\left(A_{j}\right)$, that is, $\mathbf{F} \equiv F(\mathbf{T}(p+i, i))=F(\mathbf{T}(p+$ $j, j)) \subset \bigcap_{j=1}^{p} T_{j}\left(A_{j}\right) ; \forall i, j(\neq i) \in \bar{p}$. 
(ii) There is a unique set $\left\{x_{i 1}, x_{i 2}, \ldots, x_{i p}\right\}$ satisfying the constraints $x_{i j+1}=T_{j} x_{i j} \in A_{j+1}$, subject to $x_{i p+1}=x_{i 1}$, for any given $x_{i 1} \in \mathbf{F} \subset A_{i}$ and for any given $i \in \bar{p}$. Furthermore, each of those sets satisfies the limiting property $x_{i j+1}=\left(T_{j} \circ T_{j-1} \circ \cdots \circ T_{i}\right) \circ \lim _{\ell \rightarrow \infty}\left(\mathbf{T}^{p \ell}(p+i, i)\right) x_{i} ; \forall j \in \bar{p}$ for each $x_{i} \in A_{i}$ and any given $i \in \bar{p}$.

(iii) $\mathbf{F}=\left\{z \in \bigcap_{j=1}^{p} T_{j}\left(A_{j}\right)\right\}$ consists of a unique point $z$ if $(X, d)$ is a complete metric space.

(iv) If $(X, d)$ is a complete metric space, then there is a unique set $\left\{x_{1}, x_{2}, \ldots, x_{p}\right\}$ satisfying $x_{j+1}=T_{j} x_{j}=\left(T_{j} \circ T_{j-1} \circ \cdots \circ T_{i}\right) \circ \lim _{\ell \rightarrow \infty}\left(\mathbf{T}^{p \ell}(p+i, i)\right) \omega_{i}=\lim _{\ell \rightarrow \infty}\left(\mathbf{T}^{p \ell+j}(p+i, i)\right) \omega_{i} \in A_{j+1} ;$ $\forall j \in \bar{p}$ with $x_{1}=z \in \mathbf{F}$ for any $\omega_{i} \in A_{i}$ and any given $i \in \bar{p}$.

Proof. (i) If $A_{i} \subseteq A_{i+1} ; \forall i \in \bar{p}$, then $\operatorname{dist}\left(A_{i}, A_{i+1}\right)=0$ and $\bigcap_{i=1}^{p} T_{i}\left(A_{i}\right) \subseteq \bigcap_{i=1}^{p} A_{i} \neq \emptyset$. From Proposition 3.9, constraint (3.11) holds with $k \in[0,1)$ and $\operatorname{dist}\left(A_{i}, A_{i+1}\right)=0 ; \forall i \in \bar{p}$ so that

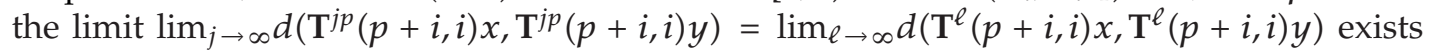
and is equal to zero; $\forall x \in A_{i}, \forall y \in A_{i+1}, \forall i \in \bar{p}$. Then, $\exists z_{i} \in F(\mathbf{T}(p+i, i)) \neq \emptyset ; \forall i \in \bar{p}$ since $F(\mathbf{T}(p+i, i)) \subset A_{i}, z_{i} \subset A_{i} ; \forall i \in \bar{p}$.

If $(X, d)$ is complete, then $F(\mathbf{T}(p+i, i))=\left\{z_{i} \in A_{i}\right\}$ since the fixed point is unique. It is now proven by contradiction that $z_{i} \in \bigcap_{j=1}^{p} A_{j} ; \forall i \in \bar{p}$. Assume that $F(\mathrm{~T}(p+i, i)) \ni z_{i} \notin \bigcap_{j=1}^{p} A_{j}$ so that $z_{i} \notin \bigcap_{j=1}^{p} T_{j}\left(A_{j}\right)$ since $\bigcap_{j=1}^{p} T_{j}\left(A_{j}\right) \subseteq \bigcap_{j=1}^{p} A_{j}$. Then, $\mathbf{T}(p+i, i) z \in \bigcap_{j=1}^{p} T_{j}\left(A_{j}\right) ; \forall z \in A_{i}$ from the definition of the composed self-mapping $\mathbf{T}(p+i, i): A_{i} \rightarrow A_{i}$ as $\mathbf{T}(p+i, i):=$ $T_{p+i} \circ T_{p+i-1} \circ \cdots \circ T_{i+1} \circ T_{i} ; \forall i \in \bar{p}$.

As a result, $F(\mathbf{T}(p+i, i)) \ni z_{i}=\mathbf{T}(p+i, i) z_{i} \in \bigcap_{j=1}^{p} T_{j}\left(A_{j}\right) \subseteq \bigcap_{j=1}^{p} A_{j}$ which is a contradiction to the above assumption and proves the result. Now, it is proven that $\mathbf{F} \equiv F(\mathbf{T}(p+i, i))=F(\mathbf{T}(p+j, j)) ; \forall i, j(\neq i) \in \bar{p}$. Proceed by contradiction. Assume that $z_{j} \in F(\mathbf{T}(p+j, j)) \cap \overline{F(\mathbf{T}(p+i, i))}$ for some $(i, j(\neq i)) \in \bar{p} \times \bar{p}$. Note that $z_{j} \in \bigcap_{\ell=1}^{p} T\left(A_{j}\right) \subseteq$ $\bigcap_{\ell=1}^{p} A_{j} \subseteq A_{i}$ for the given $i \in \bar{p}$ since $F(\mathbf{T}(p+i, i)) \subset \bigcap_{j=1}^{p} T_{j}\left(A_{j}\right) \subseteq \bigcap_{j=1}^{p} A_{j}$. Thus, $\lim _{\ell \rightarrow \infty} \mathbf{T}^{p \ell}(p+j, j) z_{j}=z_{j} \in F(\mathbf{T}(p+i, i))$ since $z_{j} \in A_{i}$ which contradicts $z_{j} \in \overline{F(\mathbf{T}(p+i, i))}$. Then, $\mathbf{F} \equiv F(\mathbf{T}(p+i, i))=F(\mathbf{T}(p+j, j))$. Property (i) has been proven.

(ii) Let $z \in \mathbf{F} \equiv F(\mathbf{T}(p+i, i))$ be a fixed point of $\mathbf{T}(p+i, i): A_{i} \cup A_{i+1} \rightarrow A_{i} \cup A_{i+1}$ for any $i \in \bar{p}$. A sequence $x_{j+1}=T_{j} x_{j} ; j=i, i+1, \ldots, i+p$ of $p$ points exists obeying the iteration

$$
\begin{aligned}
x_{i+j} & =\left(T_{i+j-1} \circ T_{i+j-2} \circ \cdots \circ T_{i}\right) z \\
& =\left(T_{i} \circ \mathbf{T}(p+i, i)\right) z \\
& =\lim _{j \rightarrow \infty}\left(T_{i+j-1} \circ T_{i+j-2} \circ \cdots \circ T_{i} \circ \mathbf{T}^{p j}(p+i, i)\right) y_{i} \\
& =\left(T_{i+j-1} \circ T_{i+j-2} \circ \cdots \circ T_{i} \circ \lim _{j \rightarrow \infty} \mathbf{T}^{p j}(p+i, i)\right) y_{i} \\
& =\left(T_{i+j-1} \circ T_{i+j-2} \circ \cdots \circ T_{i}\right) z
\end{aligned}
$$

for some $y_{i} \in A_{i}$ for any $i \in \bar{p} ; \forall j \in \bar{p}$ subject to $x_{j+p}=x_{j}$, since

$$
\mathbf{F} \ni z=\mathbf{T}(p+i, i) z=\lim _{j \rightarrow \infty} T^{p j}(p+i, i) y_{i} \quad \text { for some } y_{i} \in A_{i}, \forall i \in \bar{p}
$$


Then, the $p$-tuple $S(z)=\left(x_{1}, x_{2}, \ldots, x_{p}\right) \in A_{1} \times A_{2} \times \cdots \times A_{p}$, and thus the corresponding set $\left\{x_{1}, x_{2}, \ldots, x_{p}\right\}$ is unique for each $z \equiv x_{j} \in F(\mathrm{~T})$ and some $j \in \bar{p}$ since $T_{i}$ is a self-mapping on $A_{i} ; \forall i \in \bar{p}$. On the other hand, there exists $z_{i} \in F(\mathbf{T})$ such that $z_{i}=\mathbf{T}(p+i, i) z_{i}=\lim _{j \rightarrow \infty} T^{p j}(p+$ $i, i) y_{i}$ for each $y_{i} \in A_{i}$ and each $i \in \bar{p}$. Thus, a unique $p$-tuple $S\left(z_{i}\right)$ exists for each $z_{i} \in F(\mathbf{T})$ and then a unique set $\left\{x_{i 1}, x_{i 2}, \ldots, x_{i p}\right\} ; \forall i \in \bar{p}$. Property (ii) has been proven. Property (iii) follows directly from Property (i) together with Property (ii) since $(X, d)$ is complete. Property (iv) follows directly from Properties (ii) and (iii).

Note that only a point in the unique set $\left\{x_{1}, x_{2}, \ldots, x_{p}\right\}$, referred to in Corollary 3.10(iv), is a fixed point of the composed $p$-cyclic $k$-contraction self-mappings $\mathbf{T}(p+i, i)$ on $A_{i} \cup A_{i+1}$, $\forall i \in \bar{p}$ if the metric space $(X, d)$ is complete. Also, there is only a guaranteed fixed point of the composed $p$-cyclic $k$-contraction self-mappings $\mathbf{T}(p+i, i)$ on $A_{i} \cup A_{i+1} ; \forall i \in \bar{p}$, referred to in Corollary 3.10(ii), in each of the corresponding unique sets $\left\{x_{i 1}, x_{i 2}, \ldots, x_{i p}\right\}$ if $(X, d)$ is not complete.

An extra conclusion result can be obtained from Corollary 3.10 as follows in view of Remark 3.5:

Corollary 3.11. The images of the mappings $T^{p \ell}(p+i, i)$ are in $\bigcap_{i \in \bar{p}} \bigcap_{j \in I_{i}} T\left(A_{j}\right) ; \forall \ell \in \mathbf{Z}_{+}, \forall i \in \bar{p}$. If, furthermore, $\prod_{i=1}^{p}\left[k_{i}\right]<1$ and $A_{i} \subseteq A_{i+1} ; \forall i \in \bar{p}$, then the image of $T^{p \ell}(p+i, i)$ is in $\bigcap_{j \in \bar{p}} T\left(A_{j}\right) \subset$ $\bigcap_{j \in \bar{p}} A_{j}$ as $\ell \rightarrow \infty ; \forall i \in \bar{p}$. Also,

$$
\mathbf{F}=\bigcup_{\ell \in \bar{p}} F(\mathbf{T}(p+\ell, \ell)) \subset \bigcap_{\ell \in \bar{p}} T\left(A_{\ell}\right) \subset \bigcap_{\ell \in \bar{p}} A_{\ell}
$$

Now, the self-mapping $T: \bigcup_{j=1}^{p} A_{j} \rightarrow \bigcup_{j=1}^{p} A_{j}$ is defined as $T x=\mathbf{T}(p+i, i) x$ for each $x \in \bigcup_{j=1}^{p} A_{j}$ such that $x \in A_{i} \cup A_{i+1} \wedge x \notin A_{j}$ for some $i \in \bar{p} ; \forall j(<i) \in \bar{p}$ such that $A_{j} \neq A_{i}$. It turns out that such a mapping is a $p$-cyclic $k$-contraction if the composed self-mappings $\mathbf{T}(p+i, i)$ on $A_{i} \cup A_{i+1}$ are composed $p$-cyclic $k$-contractions. Note that there always exists a unique $i=i(x) \in \bar{p}$ for each given $x \in \bigcup_{j=1}^{p} A_{j}$ which, in addition, fulfils $x \in \bigcup_{j=i}^{p} A_{j}$ since $T\left(A_{j}\right) \subseteq A_{j+1} ; \forall j \in \bar{p}$. The following result is obtained directly from Corollaries 3.10(i) and 3.10(iv).

Corollary 3.12. Consider the self-mapping $T: \bigcup_{j=1}^{p} A_{j} \rightarrow \bigcup_{j=1}^{p} A_{j}$, subject to $T_{i}\left(A_{j}\right) \subseteq A_{j+1} ; j=i$, $i+1$, and assume that $k=\prod_{i=1}^{p}\left[k_{i}\right]<1$ and $A_{i} \subseteq A_{i+1} ; \forall i \in \bar{p}$ so that $T: \bigcup_{j=1}^{p} A_{j} \rightarrow \bigcup_{j=1}^{p} A_{j}$ is a $p$-cyclic $k$-contraction. Then, $F(\mathbf{T}) \equiv \mathbf{F}=\left\{z \in \bigcap_{j=1}^{p} T_{j}\left(A_{j}\right)\right\} \neq \emptyset$ which, furthermore, consists of a single point if $(X, d)$ is a complete metric space.

The relation between composed $p$-cyclic $k$-contractions satisfying Corollaries 3.103.12 and the so-called $p$-cyclic $(\alpha, \beta)$-Kannan self-mappings defined below is now discussed. Let $A_{i}$ be nonempty subsets of a metric space $(X, d) ; \forall i \in \bar{p}$. Consider the mappings $T_{i}: A_{i} \cup A_{i+1} \rightarrow A_{i+1} \cup A_{i+2}$ satisfying $T_{i}\left(A_{j}\right) \subseteq A_{j+1}$ for $j=i, i+1 ; \forall i \in \bar{p}$ for the nonempty subsets $A_{i}$ of the metric space $(X, d)$. Note that this implies that $\mathbf{T}(p+i, i)\left(A_{i} \cup A_{i+1}\right) \subseteq$ $A_{i} \cup A_{i+1}, \mathbf{T}(p+i, i)\left(A_{i}\right) \subseteq A_{i}$, and $\mathbf{T}(p+i, i)\left(A_{i+1}\right) \subseteq A_{i+1}$. The following definition which generalizes Definition 2.1 is then used to prove further results. 
Definition 3.13. A self-mapping $\mathbf{T}(p+i, i): A_{i} \cup A_{i+1} \rightarrow A_{i} \cup A_{i+1}$ is a composed $p$-cyclic $(\alpha, \beta)$ Kannan self-mapping if it satisfies the following property for some real $\alpha \in[0,1 / 2)$ and some $\beta \in \mathbf{R}_{+}$:

$$
\begin{aligned}
d(\mathbf{T}(p+i, i) x, \mathbf{T}(p+i, i) y) \leq & \alpha(d(x, \mathbf{T}(p+i, i) x)+d(y, \mathbf{T}(p+i, i) y)) \\
& +\beta(1-\alpha) \operatorname{dist}\left(A_{i}, A_{i+1}\right) ; \quad \forall x \in A_{i}, \forall y \in A_{i+1}
\end{aligned}
$$

Proposition 3.14. Consider the self-mappings $\mathbf{T}(p+i, i): A_{i} \cup A_{i+1} \rightarrow A_{i} \cup A_{i+1}$ with $\mathbf{T}(p+i, i)\left(A_{j}\right) \subseteq A_{j+1}$ for $j=i, i+1 ; \forall i \in \bar{p}$ being composed $p$-cyclic $k$-contractions satisfying $k=\prod_{i=1}^{p}\left[k_{i}\right] \in[0,1 / 3)$. The following properties hold.

(i) The self-mappings $\mathbf{T}(p+i, i): A_{i} \cup A_{i+1} \rightarrow A_{i} \cup A_{i+1} ; \forall i \in \bar{p}$ and $T: \bigcup_{j=1}^{p} A_{j} \rightarrow \bigcup_{j=1}^{p} A_{j}$ are $\alpha$-Kannan self-mappings with $\alpha:=k /(1-k) \in[0,1 / 2)$.

(ii) The self-mappings $\mathbf{T}(p+i, i): A_{i} \cup A_{i+1} \rightarrow A_{i} \cup A_{i+1}$ and $T: \bigcup_{j=1}^{p} A_{j} \rightarrow \bigcup_{j=1}^{p} A_{j}$ are, respectively, $p$-cyclic $(\alpha, \beta)$-Kannan self-mappings for all $i \in \bar{p}$ and composed $p$-cyclic $(\alpha, \beta)$-Kannan self-mappings for some real constant $\alpha \in[0,1 / 2)$ and any $\beta \in \mathbf{R}_{+}$.

Proof. (i) From (3.12) and the triangle inequality of the distance mapping

$$
\begin{aligned}
d(\mathbf{T}(p+i, i) x, \mathbf{T}(p+i, i) y) \leq & k d(x, y) \\
\leq & k(d(x, \mathbf{T}(p+i, i) x)+d(\mathbf{T}(p+i, i) x, \mathbf{T}(p+i, i) y) \\
& +d(y, \mathbf{T}(p+i, i) y))
\end{aligned}
$$

for a given $i \in \bar{p}$ and $\forall(x, y) \in A_{i} \times A_{i+1}$ since $A_{i} \subseteq A_{i+1} ; \forall i \in \bar{p}$. Since $k \in[0,1)$,

$$
d(\mathbf{T}(p+i, i) x, \mathbf{T}(p+i, i) y) \leq \alpha(d(x, \mathbf{T}(p+i, i) x)+d(y, \mathbf{T}(p+i, i) y))
$$

with $\alpha:=k /(1-k) \in[0,1 / 2)$ since $k \in[0,1 / 3)$ so that $\mathbf{T}(p+i, i): A_{i} \cup A_{i+1} \rightarrow A_{i} \cup A_{i+1}$ is a $\alpha$-Kannan self-mapping $\forall i \in \bar{p}$ from (2.1) and so it is $T: \bigcup_{j=1}^{p} A_{j} \rightarrow \bigcup_{j=1}^{p} A_{j}$ by construction. Property (i) has been proven. (ii) The proof follows directly since $\beta(1-\alpha) \operatorname{dist}\left(A_{i}, A_{i+1}\right) ; \forall i \in \bar{p}$ so that (3.23) implies that (3.21) holds.

Remark 3.15. It turns out that Proposition 3.14(ii) which is slightly modified still holds if the inclusion conditions $A_{i} \subseteq A_{i+1} ; \forall i \in \bar{p}$ are removed. In fact, the self-mappings $\mathbf{T}(p+i, i)$ and $T$ on $A_{i} \cup A_{i+1}$ and on $\bigcup_{j=1}^{p} A_{j}$, respectively, are $p$-cyclic $(\alpha, \beta)$-Kannan self-mappings; $\forall i \in \bar{p}$ and composed $p$-cyclic $(\alpha, \beta)$-Kannan self-mappings, respectively, for some real constant $\alpha \in[0,1 / 2)$ and $\forall \beta \geq \beta_{0}:=(1-k) /(1-2 k)$. The proof follows directly from that of Proposition 3.14(ii) and Definition 3.13 (see, in particular, (3.21)).

Definition 2.7 is generalized as follows for the case $p \geq 3$, and the subsequent theorem compares $p$-cyclic $k$-contractions with those of Meir-Keeler type. 
Definition 3.16 (see [12]). Assume that $A_{i} \subseteq A_{i+1}$ are nonempty subsets of a metric space $(X, d)$ with $A_{p+1}=A_{p}$. A $p$-cyclic self-mapping $T: \bigcup_{i=1}^{p} A_{i} \rightarrow \bigcup_{i=1}^{p} A_{i}$ is a contraction of Meir-Keeler type if for any given $\varepsilon \in \mathbf{R}_{+}, \exists \delta=\delta(\varepsilon) \in \mathbf{R}_{+}$such that

$$
\begin{array}{r}
d(x, y)<\varepsilon+\delta+\operatorname{dist}\left(A_{i}, A_{i+1}\right) \Longrightarrow \\
\quad d(T x, T y)<\varepsilon+\operatorname{dist}\left(A_{i}, A_{i+1}\right) \\
\forall(x, y) \in A_{i} \times A_{i+1}, \quad \forall i \in \bar{p}
\end{array}
$$

Note that the equivalent contrapositive logic proposition to (3.24) is

$$
\begin{array}{r}
d(T x, T y) \geq \varepsilon+\operatorname{dist}\left(A_{i}, A_{i+1}\right) \Longrightarrow d(x, y) \geq \varepsilon+\delta+\operatorname{dist}\left(A_{i}, A_{i+1}\right) \\
\forall(x, y) \in A_{i} \times A_{i+1}, \quad \forall i \in \bar{p}
\end{array}
$$

which can be used equivalently to state Definition 3.16. The following technical simple result will be then used in the proof of Theorem 3.18 below.

Assertion 1. If (3.24), and equivalently (3.25), holds for some $\delta=\delta(\varepsilon) \in \mathbf{R}_{+}$, for any given $\varepsilon \in \mathbf{R}_{+}$, then they also hold for some $\delta \in(0, \varepsilon]$.

Proof. If $\delta \in(0, \varepsilon]$ for the given $\varepsilon \in \mathbf{R}_{+}$, the result is proven. If $\delta>\varepsilon$, then (3.25) leads directly to the property

$$
d(T x, T y) \geq \varepsilon+\operatorname{dist}\left(A_{i}, A_{i+1}\right) \Longrightarrow d(x, y) \geq \varepsilon+\delta+\operatorname{dist}\left(A_{i}, A_{i+1}\right) \geq \varepsilon+\delta^{\prime}+\operatorname{dist}\left(A_{i}, A_{i+1}\right)
$$

for any $\delta^{\prime}=\delta^{\prime}(\delta(\varepsilon)) \leq \delta(\varepsilon) ; \forall(x, y) \in A_{i} \times A_{i+1}, \forall i \in \bar{p}$, and the result holds with the replacement $\delta \rightarrow \delta^{\prime}$.

Proposition 3.17. Let $T$ be a p-cyclic self-mapping on $\bigcup_{i=1}^{p} A_{i}$. Thus, if $T: \bigcup_{i=1}^{p} A_{i} \rightarrow \bigcup_{i=1}^{p} A_{i}$ is a $p$-cyclic $k$-contraction, then it is also a contraction of Meir-Keeler type

$$
\begin{gathered}
\delta \leq \varepsilon<2 k D<2 D \\
2 \delta \leq(1-k) \varepsilon+k \delta \leq D:=\max \left(\operatorname{diam}\left(A_{i}\right): i \in \bar{p}\right) \\
\leq \frac{1}{2}(\varepsilon+\delta+(1-k)(\varepsilon-\delta)) \leq \varepsilon+(1-k)(\varepsilon-\delta) .
\end{gathered}
$$

Proof. Since $T: \bigcup_{i=1}^{p} A_{i} \rightarrow \bigcup_{i=1}^{p} A_{i}$ is a $p$-cyclic $k$-contraction, then it is $p$-cyclic nonexpansive so that $d_{A}:=\operatorname{dist}\left(A_{i}, A_{i+1}\right) ; \forall i \in \bar{p}$. Take any $(x, y) \in A_{i} \times A_{i+1}$ for some $i \in \bar{p}$ such that $d(x, y)<\varepsilon+\delta+d$. Then, since $T: \bigcup_{i=1}^{p} A_{i} \rightarrow \bigcup_{i=1}^{p} A_{i}$ is a $p$-cyclic $k$-contraction, one gets that

$$
\begin{aligned}
d(T x, T y) & \leq k d(x, y)+(1-k) \operatorname{dist}\left(A_{i}, A_{i+1}\right)<k\left(\varepsilon+\delta+d_{A}\right)+(1-k) d_{A} \\
& =k(\varepsilon+\delta)+d_{A}<\varepsilon+d ; \quad \forall(x, y) \in A_{i} \times A_{i+1}, \forall i \in \bar{p}
\end{aligned}
$$


provided that for any given $\varepsilon \in \mathbf{R}_{+}, \delta=\delta(\varepsilon) \in[0,(1-k)(\varepsilon+d) / k)$ since $k \in[0,1)$. Then, the $p$ cyclic self-mapping $T$ on $\bigcup_{i=1}^{p} A_{i}$ is also a contraction of Meir-Keeler type from Definition 3.16.

The subsequent result relies on the limiting property to the best proximity points of the distances between points in adjacent sets in self-mappings being $p$-cyclic contractions of Meir-Keeler type.

Theorem 3.18. Let $T: \bigcup_{i=1}^{p} A_{i} \rightarrow \bigcup_{i=1}^{p} A_{i}$ be a p-cyclic contraction of Meir-Keeler type. Then the following properties hold.

(i) If $d(x, y) \leq \varepsilon+\delta+d_{A}$ for some real constants $\varepsilon, \delta=\delta(\varepsilon) \in \mathbf{R}_{+}$then the inequalities $d_{A} \leq$ $d\left(T^{j} x, T^{j} y\right)<\varepsilon_{j}+\delta_{j}+d_{A}$ hold for some bounded positive strictly monotone decreasing real sequences $\left\{\varepsilon_{i}\right\}_{0}^{\infty},\left\{\delta_{i}(\varepsilon)\right\}_{0}^{\infty} \forall j \in \mathbf{Z}_{0+}$ which converge to zero, with $\varepsilon_{0}=\varepsilon, \delta_{0}=\delta$, and furthermore, $d\left(T^{j} x, T^{j} y\right) \rightarrow d_{A}$ as $j \rightarrow \infty ; \forall(x, y) \in A_{i} \times A_{i+1}, \forall i \in \bar{p}$, where

$$
\begin{array}{r}
d_{A}:=\operatorname{dist}\left(A_{i}, A_{i+1}\right)=d\left(z_{i}, T z_{i}\right)=d\left(z_{j}, T^{j-i} z_{j}\right) \\
\text { for } z_{j}=T^{j-i} z_{i} \in B P_{j}(T), \quad z_{i} \in B P_{i}(T)
\end{array}
$$

for $\forall i, j \in \bar{p}$. As a result, there exists a finite $M=M(\rho, \varepsilon) \in \mathbf{Z}_{0+}$ such that

$$
d_{A} \leq d\left(T^{j} x, T^{j} y\right) \leq d_{A}+\rho ; \quad \forall(x, y) \in A_{i} \times A_{i+1}, \forall i \in \bar{p}
$$

for any given $\rho \in \mathbf{R}_{+}$and $\forall j(\geq M) \in \mathbf{Z}_{0+}$.

(ii) If $\bigcap_{i=1}^{p} A_{i} \neq \emptyset$, then $\bigcap_{i=1}^{p} A_{i} \supset F(T) \ni z=\lim _{i \rightarrow \infty} T^{i} x ; \forall x \in \bigcup_{i=1}^{p} A_{i}$, and $F(T)$ consists of a unique fixed point if $(X, d)$ is complete.

(iii) There exists some real constant $\xi \in(0,1)$ such that

$$
\sum_{j=0}^{\infty} \delta_{j}=\sum_{j=0}^{N-1} \delta_{j}+\frac{1}{1-\xi}=\varepsilon<\infty
$$

and, furthermore, $\xi=(\varepsilon-\delta) /(1+\varepsilon-\delta)$ under the constraint $\varepsilon<1$ which allows the choice $\delta_{i}<1 ; \forall i \in \mathbf{Z}_{0+}$. 
Proof. (i)-(ii) A recursion in (3.24) leads to the following recursion of implications for $d_{A}:=$ $\operatorname{dist}\left(A_{i}, A_{i+1}\right) ; \forall i \in \bar{p}$ :

$$
\begin{aligned}
d_{A} & \leq d(x, y) \\
& <\varepsilon+\delta+d_{A} \Longrightarrow d_{A} \leq d(T x, T y)<\varepsilon+d_{A}=\varepsilon_{1}+\delta_{1}+d_{A} \\
& <\varepsilon+\delta+d_{A} \Longrightarrow d_{A} \leq d\left(T^{3} x, T^{3} y\right)<\varepsilon_{2}+d_{A}=\varepsilon_{3}+\delta_{3}+d_{A} \\
& \leq \varepsilon_{2}+\delta_{2}+d_{A} \Longrightarrow d_{A} \leq d\left(T^{3} x, T^{3} y\right)<\varepsilon_{2}+d_{A}=\varepsilon_{3}+\delta_{3}+d_{A} \\
& \leq \varepsilon_{2}+\delta_{2}+d_{A} \Longrightarrow d_{A} \leq d\left(T^{j} x, T^{j} y\right)<\varepsilon_{j-1}+d_{A}=\varepsilon_{j}+\delta_{j}+d_{A} \\
& \leq \varepsilon_{j-1}+\delta_{j-1}+d_{A} \quad \forall(x, y) \in A_{i} \times A_{i+1}, \forall i \in \bar{p}
\end{aligned}
$$

for $\delta=\delta(\varepsilon) \in \mathbf{R}_{+}$for any arbitrary given $\varepsilon \in \mathbf{R}_{+}$and some positive real sequences $\left\{\delta_{i}\right\}_{0}^{\infty}$ and $\left\{\varepsilon_{i}\right\}_{0}^{\infty}$ which depend on $\varepsilon$ according to the respective implicit dependences:

$$
\delta_{i}=\delta_{i}\left(\varepsilon_{j}: j(\leq i) \in \mathbf{Z}_{0+}\right)=g_{i}(\varepsilon) ; \quad \varepsilon_{i}=\varepsilon_{i}\left(\varepsilon_{j}: j(\leq i-1) \in \mathbf{Z}_{0+}\right)=h_{i}(\varepsilon) ; \quad \forall i \in \mathbf{Z}_{0+}
$$

with $\varepsilon_{0}=\varepsilon, \delta_{0}=\delta$ which satisfy the constraints

$$
0<\delta_{i}:=\varepsilon_{i-1}-\varepsilon_{i} \leq \varepsilon_{i-1}-\varepsilon_{i}+\delta_{i-1} ; \quad \forall i \in \mathbf{Z}_{+}
$$

which imply that

$$
\begin{aligned}
0<\varepsilon_{i}<\varepsilon_{i-1}<\varepsilon_{0} & =\varepsilon<\infty ; \quad \forall i \in \mathbf{Z}_{0+} \Longrightarrow \varepsilon_{i} \longrightarrow 0, \text { as } i \longrightarrow \infty \\
0<\sum_{j=1}^{i} \delta_{j} & =\varepsilon-\varepsilon_{i}<\infty ; \quad \forall i \in \mathbf{Z}_{0+} \Longrightarrow 0<\sum_{j=1}^{\infty} \delta_{j}=\lim _{i \rightarrow \infty} \sum_{j=1}^{i} \delta_{j} \\
& =\varepsilon<\infty \Longrightarrow \delta_{i} \longrightarrow 0, \quad \text { as } i \longrightarrow \infty
\end{aligned}
$$

Furthermore, from (3.35) into (3.32), it follows that

$$
\begin{aligned}
d_{A} & \leq \inf _{(x, y) \in A_{i} \times A_{i+1}, i \in \bar{p}} \liminf _{j \rightarrow \infty} d\left(T^{j} x, T^{j} y\right) \\
& \leq \sup _{(x, y) \in A_{i} \times A_{i+1}, i \in \bar{p}} \limsup _{j \rightarrow \infty} d\left(T^{j} x, T^{j} y\right)<d_{A}+\varepsilon<\infty
\end{aligned}
$$

so that

$$
\sup _{(x, y) \in A_{i} \times A_{i+1}, i \in \bar{p}} \limsup _{j \rightarrow \infty} d\left(T^{j} x, T^{j} y\right)=d_{A}+\varepsilon_{0}<d_{A}+\varepsilon<\infty
$$


for some $\varepsilon_{0} \in[0, \varepsilon) \cap \mathbf{R}_{0+}$, which does not depend neither on the particular initial pair $(x, y) \in$ $A_{i} \times A_{i+1}, i \in \bar{p}$ nor on the given arbitrary $\varepsilon \in \mathbf{R}_{+}$since it is the supremum of all the limit superiors. Assume that $\varepsilon_{0}(\neq 0) \in \mathbf{R}_{+}$. Since $\varepsilon \in \mathbf{R}_{+}$is arbitrary, it may be chosen as $\varepsilon=\varepsilon_{0} / 2$ which contradicts $d_{A}+\varepsilon_{0}<d_{A}+\varepsilon$. Then,

$$
\begin{gathered}
\sup _{(x, y) \in A_{i} \times A_{i+1}, i \in \bar{p}} \limsup _{j \rightarrow \infty} d\left(T^{j} x, T^{j} y\right)=d_{A}, \\
d_{A} \leq \liminf _{i \rightarrow \infty} d\left(T^{i} x, T^{i} y\right) \leq \limsup _{i \rightarrow \infty} d\left(T^{i} x, T^{i} y\right)=d_{A} \Longrightarrow \exists \lim _{i \rightarrow \infty} d\left(T^{i} x, T^{i} y\right)=d_{A}, \\
\forall(x, y) \in A_{i} \times A_{i+1}, \quad \forall i \in \bar{p} .
\end{gathered}
$$

Also, since

$$
\begin{gathered}
d_{A}=\operatorname{dist}\left(A_{i}, A_{i+1}\right)=d\left(z_{i}, T z_{i}\right)=d\left(z_{j}, T^{j-i} z_{j}\right), \\
z_{j}=T^{j-i} z_{i} \in \mathrm{BP}_{j}(T), z_{i} \in \mathrm{BP}_{i}(T)
\end{gathered}
$$

for $\forall i, j \in \bar{p}$. If $d_{A}=0$, then $\mathrm{BP}_{i}(T) \equiv F(T) ; \forall i \in \bar{p}$ so that $\lim _{i \rightarrow \infty} d\left(T^{i} x, T^{i} y\right)=0, \forall(x, y) \in$ $A_{i} \times A_{i+1}, \forall i \in \bar{p}$, and since $\left(A_{i} \cap F(T)\right) \ni z=T z \in\left(A_{i+1} \cap F(T)\right)$ from $T\left(A_{i}\right) \subseteq A_{i+1} ; \forall i \in \bar{p}$, then $\bigcap_{i=1}^{p} A_{i} \supset F(T) \ni z=\lim _{i \rightarrow \infty} T^{i} x ; \forall x \in \bigcup_{i=1}^{p} A_{i}$. This conclusion is direct from the following reasoning. Assume that $F(T) \ni z \in A_{i}$ for some arbitrary $i \in \bar{p}$ which exists since $z$ is a fixed point. Then, $z=T^{j} z \in A_{i+j}$ for any $j \in \mathbf{Z}_{+}$with $A_{j+p}=A_{j} ; \forall j \in \mathbf{Z}_{+}$. Thus, $z \in \bigcap_{i=1}^{p} A_{i}$. Also, $F(T)$ consists of a unique fixed point if $(X, d)$ is complete. Properties (i) and (ii) have been proven.

It turns out from (3.35) that for some finite $\mathbf{Z}_{0+} \ni N=N(\varepsilon), \delta_{i}<1 ; \forall i \geq N$. Then, by construction, it follows that there exist some real constant $\xi_{0} \in(0,1)$ and some real constant $\xi\left(\leq \xi_{0}\right) \in(0,1)$ (both of them are dependent on $N$ and $\varepsilon$ ) which are the respective ratios of the geometric series $\sum_{j=N}^{\infty} \xi_{0}^{j}$ and $\sum_{j=0}^{\infty} \xi^{j}$, such that the following identities hold for any given sequence $\left\{\delta_{i}\right\}_{0}^{\infty}$ that satisfies (3.35):

$$
\begin{aligned}
\sum_{j=N}^{\infty} \delta_{j} & =\sum_{j=N}^{\infty} \xi_{0}^{j}=\sum_{j=0}^{\infty} \xi^{j}=\frac{\xi_{0}^{N}}{1-\xi_{0}}=\frac{1}{1-\xi} \leq \varepsilon<\infty, \\
\sum_{j=0}^{\infty} \delta_{j} & =\sum_{j=0}^{N-1} \delta_{j}+\sum_{j=N}^{\infty} \delta_{j}=\sum_{j=0}^{N-1} \delta_{j}+\sum_{j=N}^{\infty} \xi_{0}^{j}=\sum_{j=0}^{N-1} \delta_{j}+\frac{\xi_{0}^{N}}{1-\xi_{0}} \\
& =\sum_{j=0}^{N-1} \delta_{j}+\sum_{j=0}^{\infty} \xi^{j}=\sum_{j=0}^{N-1} \delta_{j}+\frac{1}{1-\xi}=\varepsilon<\infty .
\end{aligned}
$$

If $\varepsilon<1$, then a sequence satisfying $\delta_{i}<1 ; \forall i \in \mathbf{Z}_{0+}$ is valid from Assertion 1 . Thus, $N=0$, and since $\delta$ may be always taken as on being larger than $\varepsilon$, then

$$
\delta+\sum_{j=1}^{\infty} \delta_{j}=\delta+\sum_{j=1}^{\infty} \xi^{j}=\delta+\frac{\xi}{1-\xi}=\varepsilon<\infty \Longleftrightarrow \xi=\frac{\varepsilon-\delta}{1+\varepsilon-\delta}
$$


and together with (3.38), it follows that $2 \varepsilon_{i} \geq \varepsilon_{i}+\delta_{i} \geq \delta ; \forall i \in \mathbf{Z}_{+}$since

$$
\varepsilon_{i+1}=\varepsilon-\delta_{i+1}-\sum_{j=1}^{i} \delta_{j} \geq \varepsilon-\delta_{i+1}-\sum_{j=1}^{\infty} \delta_{j} \geq \varepsilon-\delta_{i+1}-\frac{\xi}{1-\xi}=\delta-\delta_{i+1}, \quad \forall i \in \mathbf{Z}_{0+}
$$

under the constraints $\varepsilon<1$ and $\delta_{i}<1 ; \forall i \in \mathbf{Z}_{0+}$. Property (iii) has been proven.

It is interesting to discuss when the composed self-mappings $\mathbf{T}(p+i, i)$ on $A_{i} \cup A_{i+1}$ for set-depending self-mappings $T_{j}: A_{j} \cup A_{j+1} \rightarrow A_{j+1} \cup A_{j+2} ; i, j \in \bar{p}$ as well as the selfmappings $T$ on $\bigcup_{i=1}^{p} A_{i}$ defined by $T x=\mathbf{T}(p+i, i) x$ are guaranteed to be $p$-cyclic Meir-Keeler contractions without requiring that the property holds for each individual $T_{i}: A_{i} A_{j+1} A_{i+1} \rightarrow$ $A_{i+1} A_{j+1} A_{i+2}$ subject to $A_{i+p} \equiv A_{i} \in\left\{A_{1}, A_{2}, \ldots, A_{p}\right\}$. For the related discussion, assume that $\alpha_{j} \leq d\left(T^{j+i} x, T^{j+i} y\right) \leq \beta_{j} ; \forall j \in \bar{p}, \forall i \in \mathbf{Z}_{0+}$ for a set of real constants $\alpha_{i}, \beta_{i}\left(\geq \alpha_{i}\right) \in \mathbf{R}_{0+} ; \forall i \in \bar{p}$. A direct calculation on $p$ iterations yields directly

$$
\begin{aligned}
\max \left(d_{A}, d(\mathbf{T}(i, \ell) x, \mathbf{T}(i, \ell) y)-\sum_{j=1}^{p}\left(\beta_{j}-\alpha_{j}\right)\right) \\
\leq d(\mathbf{T}(p+i, \ell) x, \mathbf{T}(p+i, \ell) y) \\
\leq d(\mathbf{T}(i, \ell) x, \mathbf{T}(i, \ell) y) \\
\quad+\sum_{j=1}^{p}(d(\mathbf{T}(j+i, \ell) x, \mathbf{T}(j+i, \ell) y)-d(\mathbf{T}(j+i-1, \ell) x, \mathbf{T}(j+i-1, \ell) y)) \\
\leq d(\mathbf{T}(i, \ell) x, \mathbf{T}(i, \ell) y)+\sum_{j=1}^{p}\left(\beta_{j}-\alpha_{j}\right) ; \quad \forall(x, y) \in A_{\ell} \times A_{\ell+1}, \forall \ell \in \bar{p}, \forall i \in \mathbf{Z}_{0+} .
\end{aligned}
$$

If the self-mappings $T_{i} \equiv T: A_{i} \cup A_{i+1} \rightarrow A_{i+1} \cup A_{i+2}$ are identical; $\forall i \in \bar{p}$, then (3.43) becomes in particular:

$$
\begin{aligned}
& \max \left(d_{A}, d\left(T^{i} x, T^{i} y\right)-\sum_{j=1}^{p}\left(\beta_{j}-\alpha_{j}\right)\right) \\
& \quad \leq d\left(T^{i+p} x, T^{i+p} y\right) \leq d\left(T^{i} x, T^{i} y\right)+\sum_{j=1}^{p}\left(d\left(T^{j} x, T^{j} y\right)-d\left(T^{i} x, T^{i} y\right)\right) \\
& \quad \leq d\left(T^{i} x, T^{i} y\right)+\sum_{j=1}^{p}\left(\beta_{j}-\alpha_{j}\right) ; \forall(x, y) \in A_{\ell} \times A_{\ell+1}, \quad \forall \ell \in \bar{p}, \forall i \in \mathbf{Z}_{0+} .
\end{aligned}
$$

The following result holds directly from (3.43) and (3.44) and Theorem 3.18. 
Theorem 3.19. The composed self-mappings $\mathbf{T}(p+i, i)$ on $A_{i}$ for set-depending self-mappings $T_{j}$ : $A_{j} \cup A_{j+1} \rightarrow A_{j+1} \cup A_{j+2} ; i, j \in \bar{p}$ as well as the self-mappings $T$ on $\bigcup_{i=1}^{p} A_{i}$ defined by $\mathrm{T} x=\mathbf{T}(p+i, i) x$ are guaranteed to be as follows.

(a) $p$-cyclic Meir-Keeler contractions if $\sum_{j=1}^{p}\left(\beta_{j}-\alpha_{j}\right)<0$,

Thus, there is an asymptotic convergence from any initial point to the best proximity point in general and to a fixed point if the sets in $\left\{A_{1}, A_{2}, \ldots, A_{p}\right\}$ have a nonempty intersection. The fixed point is unique if $(X, d)$ is complete.

(b) Nonexpansive p-cyclic self-mappings if $\sum_{j=1}^{p}\left(\beta_{j}-\alpha_{j}\right) \leq 0$.

(c) Expansive p-cyclic self-mappings if $\sum_{j=1}^{p}\left(\beta_{j}-\alpha_{j}\right)>0$.

More general conditions than the Meir-Keeler ones guaranteeing that the composed self-mappings $\mathbf{T}(p+i, i)$ on $A_{i} \cup A_{i+1}, i \in \bar{p}$ are asymptotic contractions are now discussed.

Theorem 3.20. Assume that there is a real sequence $\left\{\delta_{i}\right\}_{1}^{\infty}$ of finite sum $\delta \in \mathbf{R}_{+}$which satisfies the conditions

$$
\begin{gathered}
0<\delta:=\sum_{j=1}^{\infty} \delta_{j}<\infty, \\
\varepsilon+\sum_{j=i}^{\infty} \delta_{j}>0, \quad \delta_{i}>0 ; \quad \forall i(\geq N) \in \mathbf{Z}_{+}
\end{gathered}
$$

for some given real constant $\varepsilon \in \mathbf{R}_{+}$, whose elements are defined in such a way that the composed self-mapping $\mathbf{T}(i, 1)$ on satisfies

$$
\begin{array}{r}
d_{A} \leq d(\mathbf{T}(i, 1) x, \mathbf{T}(i, 1) y)<d_{A}+\varepsilon+\sum_{j=i}^{\infty} \delta_{j}=d_{A}+\varepsilon+\delta-\sum_{j=1}^{i-1} \delta_{j} \\
\forall(x, y) \in A_{\ell} \times A_{\ell+1}, \quad \forall \ell \in \bar{p}, \quad \forall i \in Z_{+}
\end{array}
$$

Then, $\exists \lim _{i \rightarrow \infty} d(\mathbf{T}(i, 1) x, \mathbf{T}(i, 1) y)=d_{A} ; \forall i \in \bar{p}$.

Proof. From the properties of the $\left\{\delta_{i}\right\}_{1}^{\infty}$ sequence and (3.46), one gets

$$
\begin{array}{r}
d(\mathbf{T}(i+1,1) x, \mathbf{T}(i+1,1) y)<d_{A}+\varepsilon+\sum_{j=i+1}^{\infty} \delta_{j}=d_{A}+\varepsilon+\delta-\sum_{j=1}^{i} \delta_{j}<d_{A}+\varepsilon \\
\forall(x, y) \in A_{\ell} \times A_{\ell+1}, \quad \forall \ell \in \bar{p} \forall i(\geq N) \in \mathbf{Z}_{+}
\end{array}
$$

for some sufficiently large finite $N=N(\varepsilon)$. The constraint on the upperbounds in (3.47) guarantees that the strict upper-bound $d_{A}+\varepsilon+\sum_{j=i+1}^{\infty} \delta_{j}$ for $d(\mathbf{T}(i+1,1) x, \mathbf{T}(i+1,1) y)$ is less 
Fixed Point Theory and Applications

than a strict upper-bound for $d(\mathbf{T}(i, 1) x, \mathbf{T}(i, 1) y)$ for any sufficiently large $i \in \mathbf{Z}_{+}$provided that

$$
\sum_{j=1}^{i} \delta_{j}=\delta_{i}+\sum_{j=1}^{i-1} \delta_{j}>\sum_{j=1}^{i-1} \delta_{j} \Longrightarrow \delta_{i}>0 ; \quad \forall i(\geq N) \in \mathbf{Z}_{+} .
$$

Furthermore, (3.46) holds $\forall i \in \mathbf{Z}_{+}$if $\varepsilon+\sum_{j=i}^{\infty} \delta_{j}>0 ; \forall i \in \mathbf{Z}_{+}$. As a result, $d(\mathbf{T}(i, 1) x, \mathbf{T}(i, 1) y) \rightarrow$ $d_{A}$ as $i \rightarrow \infty$ since the supremum of all limits superior converge to $d_{A}$ (see the proof of Theorem 3.19).

Theorem 3.20 may be particularized to $p$-cyclic asymptotic contractions as follows.

Theorem 3.21. Assume that there is a real sequence $\left\{\delta_{i}\right\}_{1}^{\infty}$ of finite sum $\delta \in \mathbf{R}_{+}$which satisfies the following conditions for some $i \in \bar{p}$ :

$$
\begin{aligned}
& 0<\delta(i):=\sum_{j=i}^{\infty} \delta_{j}<\infty, \quad \varepsilon+\delta(i)>0, \\
& \varepsilon+\sum_{j=n p+i}^{\infty} \delta_{j}>0, \quad \delta_{n p+i}>0 ; \quad \forall n(\geq N) \in \mathbf{Z}_{+}
\end{aligned}
$$

for some given real constant $\varepsilon \in \mathbf{R}_{+}$whose elements are defined in such a way that the composed self-mapping satisfies

$$
\begin{aligned}
d_{A} & \leq d(\mathbf{T}(n p+i, i) x, \mathbf{T}(n p+i, i) y)<d_{A}+\varepsilon+\sum_{j=n p+i}^{\infty} \delta_{j} \\
& =d_{A}+\varepsilon+\delta(i)-\sum_{j=i}^{n p+i-1} \delta_{j} ; \quad \forall(x, y) \in A_{i} \times A_{i+1} \text { for the given } i \in \bar{p} .
\end{aligned}
$$

Then, $\exists \lim _{n \rightarrow \infty} d(\mathbf{T}(n p+i, i) x, \mathbf{T}(n p+i, i) y)=d_{A}$ for the given $i \in \bar{p}$.

Proof. From the properties of the $\left\{\delta_{i}\right\}_{1}^{\infty}$ sequence and (3.50), one gets.

$$
\begin{aligned}
& d(\mathbf{T}(n p+i+1, i) x, \mathbf{T}(n p+i+1, i) y) \\
& <d_{A}+\varepsilon+\sum_{j=n p+i+1}^{\infty} \delta_{j}=d_{A}+\varepsilon+\delta-\sum_{j=i}^{n p+i} \delta_{j}<d_{A}+\varepsilon ; \\
& \forall(x, y) \in A_{i} \times A_{i+1} \quad \text { for the given } i \in \bar{p}, \forall n(\geq N) \in \mathbf{Z}_{0+}
\end{aligned}
$$

for some sufficiently large finite $N=N(\varepsilon, i)$ and the given $i \in \bar{p}$. The constraint on the upperbounds in (3.51) guarantees that the strict upper-bound $d_{A}+\varepsilon+\sum_{j=n p+i+1}^{\infty} \delta_{j}$ for $d(\mathbf{T}(n p+i+$ 
$1, i) x, \mathbf{T}(n p+i+1, i) y)$ is less than a strict upper-bound for $d(\mathbf{T}(n p+i, 1) x, \mathbf{T}(n p+i, 1) y)$ for any sufficiently large $n \in \mathbf{Z}_{+}$provided that

$$
\sum_{j=i}^{n p+i} \delta_{j}=\delta_{n p i}+\sum_{j=i}^{n p+i-1} \delta_{j}>\sum_{j=i}^{n p+i-1} \delta_{j} \Longrightarrow \delta_{n p+i}>0 ; \quad \forall n(\geq N) \in \mathbf{Z}_{+} \text {for the given } i \in \bar{p}
$$

Furthermore, (3.50) holds $\forall n \in \mathbf{Z}_{+}$for the given $i \in \bar{p}$ if $\varepsilon+\sum_{j=n p+i}^{\infty} \delta_{j}>0 ; \forall n \in \mathbf{Z}_{+}$for the given $i \in \bar{p}$. As a result, $d(\mathbf{T}(n p+i, i) x, \mathbf{T}(n p+i, i) y) \rightarrow d_{A}$ as $n \rightarrow \infty$ since the supremum of all limit superiors converges to $d_{A}$ (see the proof of Theorem 3.19).

Note that Theorem 3.21 guarantees that the self-mapping $\mathbf{T}(p+i, i)$ on $A_{i} \cup A_{i+1}$ has a $p$-cyclic Meir-Keeler asymptotic contraction for a particular $i \in \bar{p}$, while Theorem 3.20 guarantees that all the self-mappings $\mathbf{T}(p+i, i)$ are asymptotic contractions. In both cases, the self-mappings can be locally expansive in the sense that it can happen that $d(\mathbf{T}(j+1, i) x, \mathbf{T}(j+$ $1, i) y)>d(\mathbf{T}(j, i) x, \mathbf{T}(j, i) y)$ for some finite $j(\geq i), i \in \mathbf{Z}_{0+}$, some $(x, y) \in A_{\ell} \times A_{\ell+1}$, and some $\ell \in \bar{p}$.

\section{Acknowledgments}

The author is grateful to the Spanish Ministry of Education for its partial support to this work through Grant DPI 2009-07197. He is also grateful to the Basque Government for its support through Grants GIC07143-IT-269-07and SAIOTEK S-PE07UN04. The author thanks the reviewers for their useful comments who helped him to improve the former versions of the manuscript.

\section{References}

[1] T. Dominguez Benavides and S. Phothi, "The fixed point property under renorming in some classes of Banach spaces," Nonlinear Analysis: Theory, Methods EApplications, vol. 72, no. 3-4, pp. 1409-1416, 2010.

[2] T. Suzuki, "A new type of fixed point theorem in metric spaces," Nonlinear Analysis: Theory, Methods $\mathcal{E}$ Applications, vol. 71, no. 11, pp. 5313-5317, 2009.

[3] Dž. Burgić, S. Kalabušić, and M. R. S. Kulenović, "Global attractivity results for mixed-monotone mappings in partially ordered complete metric spaces," Fixed Point Theory and Applications, vol. 2009, Article ID 762478, 17 pages, 2009.

[4] J. J. Nieto, R. L. Pouso, and R. Rodríguez-López, "Fixed point theorems in ordered abstract spaces," Proceedings of the American Mathematical Society, vol. 135, no. 8, pp. 2505-2517, 2007.

[5] M. O. Olatinwo, "Some common fixed point theorems for selfmappings satisfying two contractive conditions of integral type in a uniform space," Central European Journal of Mathematics, vol. 6, no. 2, pp. 335-341, 2008.

[6] V. Azhmyakov, "Convexity of the set of fixed points generated by some control systems," Journal of Applied Mathematics, vol. 2009, Article ID 291849, 14 pages, 2009.

[7] M. De la Sen, "Total stability properties based on fixed point theory for a class of hybrid dynamic systems," Fixed Point Theory and Applications, vol. 2009, Article ID 826438, 19 pages, 2009.

[8] M. De la Sen, "About robust stability of dynamic systems with time delays through fixed point theory," Fixed Point Theory and Applications, vol. 2008, Article ID 480187, 20 pages, 2008.

[9] M. De la Sen, "Quadratic stability and stabilization of switched dynamic systems with uncommensurate internal point delays," Applied Mathematics and Computation, vol. 185, no. 1, pp. 508-526, 2007.

[10] M. De la Sen, "On the robust adaptive stabilization of a class of nominally first-order hybrid systems," IEEE Transactions on Automatic Control, vol. 44, no. 3, pp. 597-602, 1999. 
[11] A. Meir and E. Keeler, "A theorem on contraction mappings," Journal of Mathematical Analysis and Applications, vol. 28, no. 2, pp. 326-329, 1969.

[12] S. Karpagam and S. Agrawal, "Best proximity point theorems for $p$-cyclic Meir-Keeler contractions," Fixed Point Theory and Applications, vol. 2009, Article ID 197308, 9 pages, 2009.

[13] C.-M. Chen and T.-H. Chang, "Fixed point theorems for a weaker Meir-Keeler type $\psi$-set contraction in metric spaces," Fixed Point Theory and Applications, vol. 2009, Article ID 129124, 8 pages, 2009.

[14] A. A. Eldred and P. Veeramani, "Existence and convergence of best proximity points," Journal of Mathematical Analysis and Applications, vol. 323, no. 2, pp. 1001-1006, 2006.

[15] M. Kikkawa and T. Suzuki, "Some similarity between contractions and Kannan mappings," Fixed Point Theory and Applications, vol. 2008, Article ID 649749, 8 pages, 2008.

[16] Y. Enjouji, M. Nakanishi, and T. Suzuki, “A generalization of Kannan's fixed point theorem," Fixed Point Theory and Applications, vol. 2009, Article ID 192872, 10 pages, 2009.

[17] M. De la Sen, "Some combined relations between contractive mappings, Kannan mappings, reasonable expansive mappings, and T-stability," Fixed Point Theory and Applications, vol. 2009, Article ID 815637, 25 pages, 2009.

[18] G. Inoue, W. Takahashi, and K. Zembayashi, "Strong convergence theorems by hybrid methods for maximal monotone operators and relatively nonexpansive mappings in Banach spaces," Journal of Convex Analysis, vol. 16, no. 3-4, pp. 791-806, 2009.

[19] K. Aoyama, F. Kohsaka, and W. Takahashi, "Strongly relatively nonexpansive sequences in Banach spaces and applications," Journal of Fixed Point Theory and Applications, vol. 5, no. 2, pp. 201-225, 2009.

[20] C. Chen and C. Zhu, "Fixed point theorems for $n$ times reasonable expansive mapping," Fixed Point Theory and Applications, vol. 2008, Article ID 302617, 6 pages, 2008. 